\title{
CHIASMA DISTRIBUTION AT DIPLOTENE IN A LOCUST
}

\author{
S. A. HENDERSON \\ Agricultural Reseorch Council Unit of Biometrical Genetics, \\ Deportment of Genetics, University of Birminghom *
}

Received I4.xi.62

\section{THE PROBLEM}

THE body of supporting evidence is now so large that the correspondence of crossing over and chiasma formation is widely accepted. In the fields of genetics and cytology, respectively, they are of considerable importance yet much remains unknown of the factors which govern both the formation and the distribution of chiasmata or points of crossing over. The mechanism of formation can best be approached from a cytological standpoint and the torsional theory of Darlington $(\mathrm{r} 935 a, b)$ commands acceptance, if sometimes tentatively, by many, in preference to the alternative possibility of synthetic error or "copying-choice" (Belling, I931, 1933, Pritchard, 1960). One reason for this is the obvious inability of the "copying-choice" mechanism to account for all the observed modes of chromosome behaviour. Thus, to explain three and four strand double crossing over, additional sister-strand crossing over must be invoked, which must involve some breakage and reunion. Furthermore, "copyingchoice " can provide no explanation of the phenomenon of interference (see below).

The problem of distribution, however, may be approached from both the genetical and cytological viewpoints. From the early days of genetical research careful investigations revealed that cross-overs were not random in distribution : there was a measure of cross-over interference. One cross-over interfered with the chance of another taking place in its immediate vicinity and this decreased in intensity with increasing distance from the original cross-over (Muller, I9I6). Furthermore, overall cross-over maps showed regions of high and low crossing over along the chromosomes : cross-overs were grouped in certain regions of high density, while there were intermediate regions of low cross-over frequency (Mather, 1936).

From the cytological standpoint Haldane (I93 I) pointed out that chiasma frequency distribution was non-random and that chiasma interference must exist, for chiasma frequency data do not fit a Poisson distribution. But the most complete work undertaken on this problem is that of Mather (1936, 1937, 1938, 1940). Mainly from a consideration of chiasma frequency/chromosome length relationships, he defined the chiasma properties of bivalents in terms of two parameters, the differential (' $d$ ') distance and the interference (' $i$ ') distance.

* Present address : Department of Genetics, The University, Cambridge. 
These two variables, acting together, were responsible for determining both the frequency and position of chiasmata for each bivalent, depending on its length. The first chiasma formed a certain distance away from a fixed point on the chromosome, such as the centromere. This was the ' $d$ ' distance and was bivalent specific. Subsequent chiasmata formed at ' $i$ ' distance intervals along the chromosomes if they were sufficiently long. In view of the commonly observed linearity of chiasma frequency/chromosome length graphs, it was suggested that the ' $i$ ' distance was constant for all members of the complement.

During the past twenty years little or no additional information has been presented from any source to change or substantiate the schema suggested by Mather. Furthermore, no extensive measurements of chiasma distribution along chromosomes have ever been made to clarify these relationships. The only previous attempt at measuring chiasma positions was made in 193 I by Belling, with little success. The present investigation was undertaken to see how much light actual measurements of the positions of chiasmata along bivalents can throw on this problem. They were also desirable in order to have an accurate picture of the normal, control distribution of chiasmata in this species, for comparison with the shift in both chiasma frequency and position which is obtained at high temperatures (Henderson, 1962, 1963).

The species used in this investigation was the desert locust Schistocerca gregaria. This was chosen because it is one of the few species known which possesses all those features desirable, indeed indispensable, if such an analysis is to be attempted (see John and Henderson, 1962). These attributes are :-

I. The chromosomes are large in size, comparatively few in number and of a wide size range. Furthermore, a consistent classification of all members of the complement is possible.

2. Diplotene in male meiosis offers an unparalleled opportunity for the study of both chiasma frequency and position.

3. Chiasma movement during diplotene is not marked.

4. Throughout diplotene the positions of the centromeres in these acrocentric chromosomes are revealed by precocious procentric condensation.

With these four features it is possible to identify both ends of each chromosome within the complement and to measure the positions of chiasmata in consistent linear order along each bivalent from a fixed point of reference such as the centromere, knowing that they have not undergone considerable movement along the chromosomes.

\section{THE APPROACH}

The locusts used for this study were derived from a relatively inbred culture, started in 1946, and supplied by courtesy of the Anti-Locust 
Research Centre, London. A more detailed history of this culture is provided by John and Naylor ( ${ }_{96}^{6} \mathrm{r}$ ). Squash preparations were made of testicular follicles fixed in $1: 3$ acetic-alcohol, staining with acetic orcein.

One hundred cells at early and mid-diplotene were selected from a total of twelve individuals. All cells were photographed and each negative enlarged to a magnification of $\times 2500$ on to a sheet of white typing paper. The bivalents were drawn to an accuracy of $c$. I $\mathrm{mm}$. $(=0.4 \mu)$ and the position of the centromeric marker indicated. The ' $\mathrm{d}$ ' and ' $\mathrm{i}$ ' distances of each bivalent were then measured to the nearest I mm. $(=0 \cdot 4 \mu)$. The ' $\mathrm{d}$ ' distance was recorded as the distance from the centromere to the first chiasma and successive ' $i$, distances (' $i_{1}$ ', ' $i_{2}$ ', ' $i_{3}$ ') in linear order away from the centromere. The distance between the last chiasma and the end of the chromosome was also recorded as the remainder (' $r$ ') distance. Each one of the several thousand measurements so obtained was converted to micra and percentages of chromosome length and group and overall means calculated.

\section{THE FINDINGS}

The mean measurements obtained in the above manner for each of the eleven bivalents present within the complement, when different numbers of chiasmata are present, are provided in table I. It can be seen that the mean ' $d$ ', ' $i$ ' and ' $r$ ' distances shown in this table differ considerably from bivalent to bivalent, depending both on chiasma frequency and chromosome length.

The overall mean ' $d$ ', ' $i$ ' and ' $r$ ' values for each bivalent, irrespective of chiasma frequency, are given in table 2 . It is evident that the final ' $\bar{i}$ ' so obtained is not constant throughout the complement but increases with increase of chromosome length, increasing from $4.00 \mu$ in the case of the $\mathrm{S}_{\text {I I }}$ to $7.58 \mu$ for the LI. Indeed, the most similar distances obtained in this manner are the ' $d$ ' and ' $r$ ' distances, which is the reverse of that expected from initial hypothesis.

One point is immediately apparent from the values provided in table I. When more than one chiasma is present, these tend to be equidistantly spaced along each bivalent. When only one chiasma is present this tends to be submedian or distal in position, never strictly proximal. This is shown more clearly in fig. I, where the mean measurements in $\mu$ from table I are plotted in "bivalentdiagram" form. This equidistant spacing of chiasmata along each bivalent, irrespective of size, results in there being a very close similarity between the chiasma distributions in all bivalents for each particular chiasma frequency type. This point is emphasised by considering the relative distributions shown as percentages in table I and provided in "bivalent-diagram" form in fig. 2. Conversion to percentages 


\begin{tabular}{|c|c|c|c|}
\hline 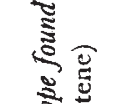 & \multicolumn{2}{|c|}{ 胥志 } & 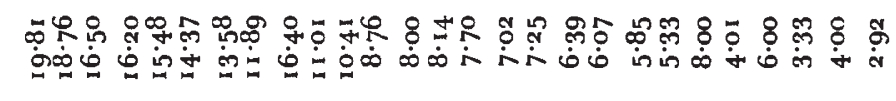 \\
\hline 离范 & \multicolumn{2}{|c|}{$\approx$} & 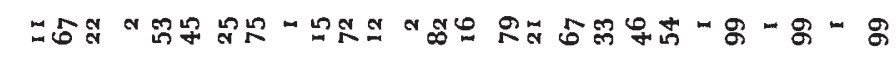 \\
\hline 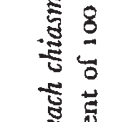 & \multirow{2}{*}{$\vdots$} & 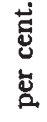 & 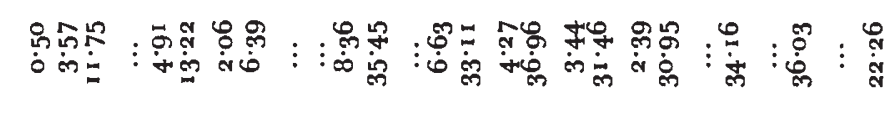 \\
\hline 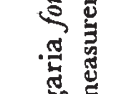 & & x' & 唡市 \\
\hline 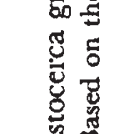 & \multirow{2}{*}{$\because$} & $\begin{array}{l}\dot{\vec{g}} \\
\dot{\mathrm{u}}\end{array}$ & 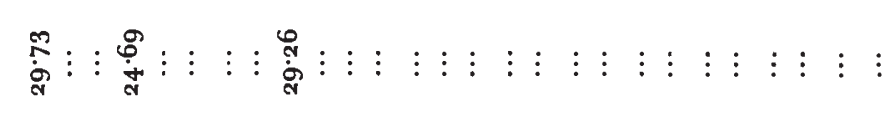 \\
\hline 造 & & is & 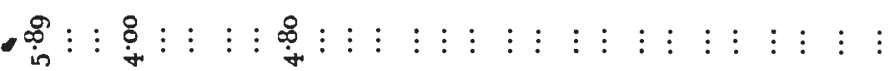 \\
\hline 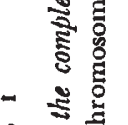 & \multirow{2}{*}{$\because$} & 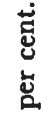 & 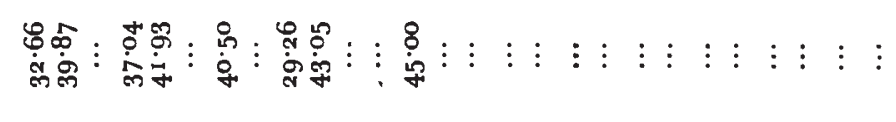 \\
\hline 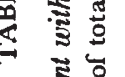 & & is & 秥 \\
\hline 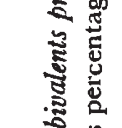 & \multirow{2}{*}{$\because$} & 递 & 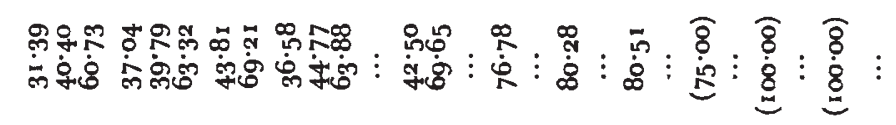 \\
\hline 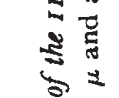 & & ise & 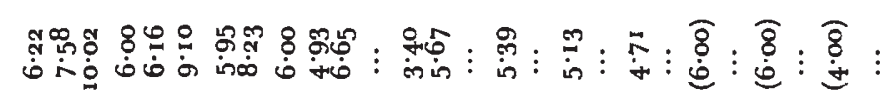 \\
\hline 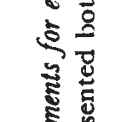 & \multirow{2}{*}{ ¿ } & 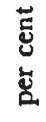 & 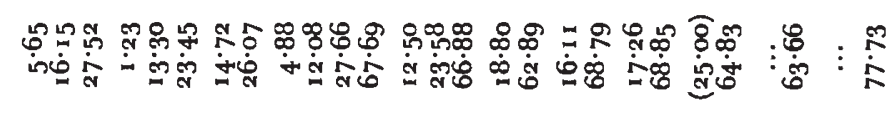 \\
\hline $\begin{array}{l}0 \\
0 \\
0 \\
0\end{array}$ & & 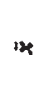 & ج \\
\hline 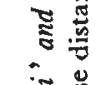 & \multicolumn{2}{|c|}{ வ } & サna toma ma ran \\
\hline$\ddot{\Sigma}$ & \multicolumn{2}{|c|}{ 总 } & 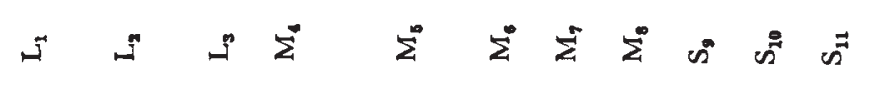 \\
\hline
\end{tabular}


removes the differences in chromosome length, thereby facilitating direct comparisons of relative positions.

There is also a positive correlation between the absolute chromosome length of each bivalent and its chiasma frequency. This can

\begin{tabular}{|c|c|c|c|c|c|c|}
\hline & $4 \times t a$. & & $3 \times 1 a$ & & $2 \times t a$. & $1 \times a$. \\
\hline$L_{1}$ & $\Longrightarrow$ & $\Longrightarrow$ & 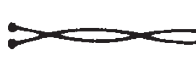 & & $\Longrightarrow$ & · \\
\hline$L_{2}$ & 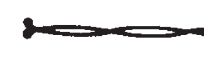 & 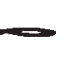 & 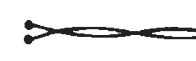 & & $\Longrightarrow$ & $\cdot$ \\
\hline$L_{3}$ & $\cdot \cdot$ & . & $\infty$ & & $\Longrightarrow$ & $\cdot \cdot$ \\
\hline$M_{4}$ & 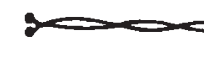 & $\infty$ & 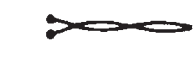 & & 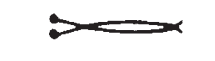 & $\Longrightarrow$ \\
\hline$M_{5}$ & $\cdot$. & - & $\infty$ & & $\infty$ & $\Rightarrow$ \\
\hline$M_{6}$ & $\cdot \cdot \cdot$ & . & $\cdot \cdot \cdot$ & & $\infty$ & $\Rightarrow$ \\
\hline$M_{7}$ & $\cdot \cdot \cdot$ & - & $\cdot \cdot \cdot$ & - & $\infty$ & $\Rightarrow$ \\
\hline$M_{8}$ & $\cdot \cdot \cdot$ & • & $\cdot \cdot$ & - & $\infty$ & 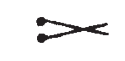 \\
\hline $\mathrm{Sg}_{9}$ & $\cdot \cdot \cdot$ & • & $\cdot \cdot$ & - & : & $\infty$ \\
\hline$S_{10}$ & $\cdot \cdot$ & . & .. & . & $8 \div \cdots$ & $>$ \\
\hline$S_{11}$ & $\cdot \cdot$ & $\cdot$ & $\cdot \cdot$ & & $8 \cdots$ & $x$ \\
\hline
\end{tabular}

FIG. I. - Mean positions of chiasmata in each of the I I bivalents of $S$. gregaria represented in "bivalent-diagram" form from the values in $\mu$ provided in table 1 . Centromeric positions marked by black circles. (The dotted figures are based on single observations).

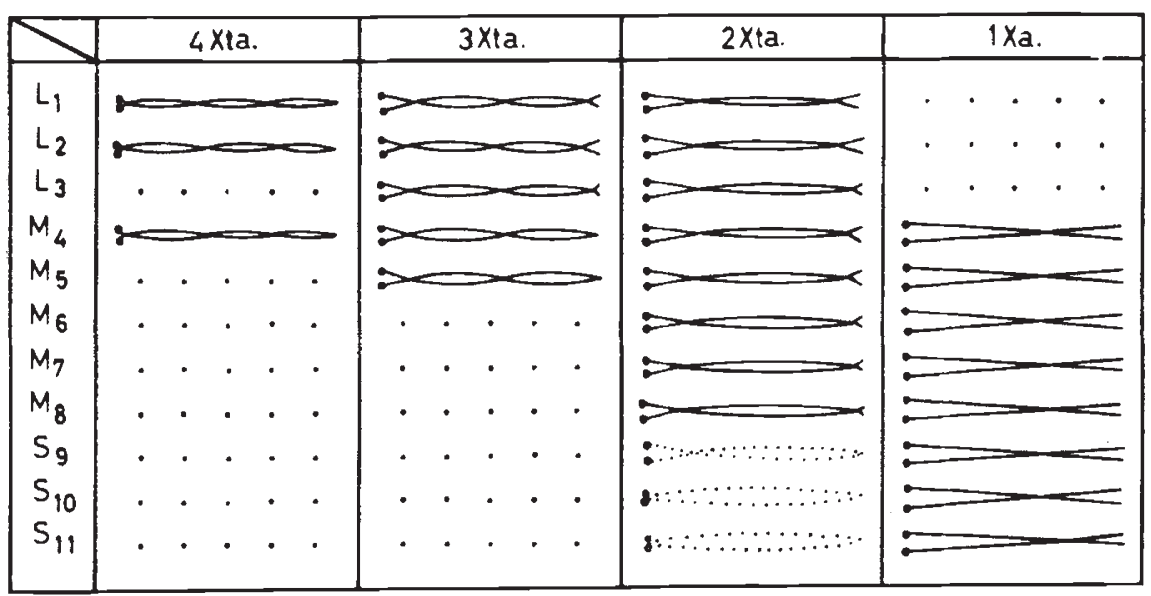

Fig. 2.- Relative positions of chiasmata in each of the II bivalents, represented in " bivalent-diagram" form from the percentages provided in table 1 .

be seen from the last column of table $\mathrm{I}$. Thus, when a particular bivalent possesses four chiasmata it is usually longer than when it has three or less, when it has three it is generally longer than when it has one or two, and bivalents possessing two chiasmata are generally longer than those possessing one. The significance of this correlation is not easy to explain. It may be that if a chromosome is longer 


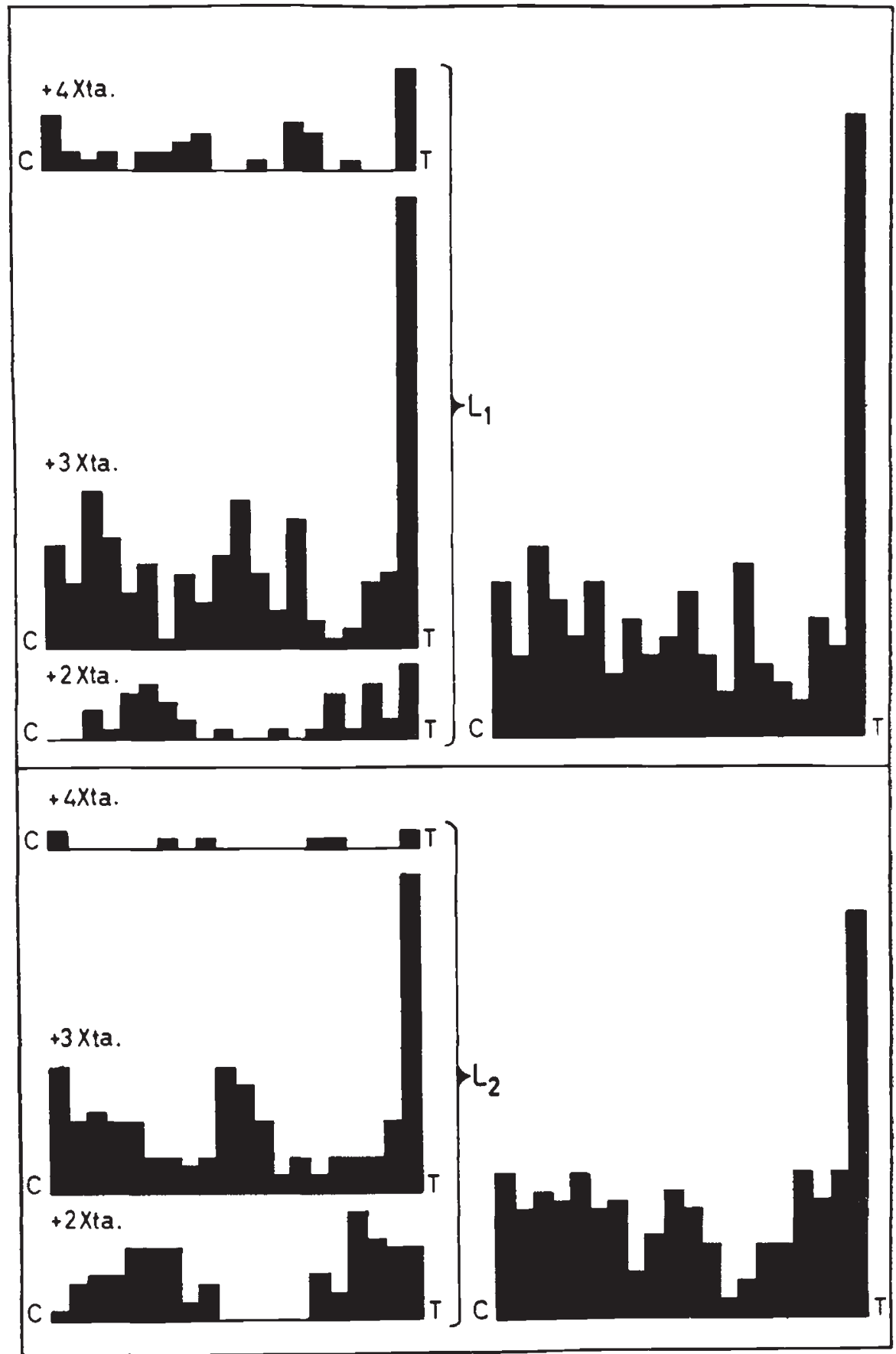

FIgs. 3-4.- Chiasma distributions for the $L_{1}$ and $L_{2}$ bivalents. In addition to the distributions for each chiasma frequency type, a composite histogram is provided to show the overall chiasma distribution $(\mathrm{C}=$ centromere, $\mathrm{T}=$ telomere). 


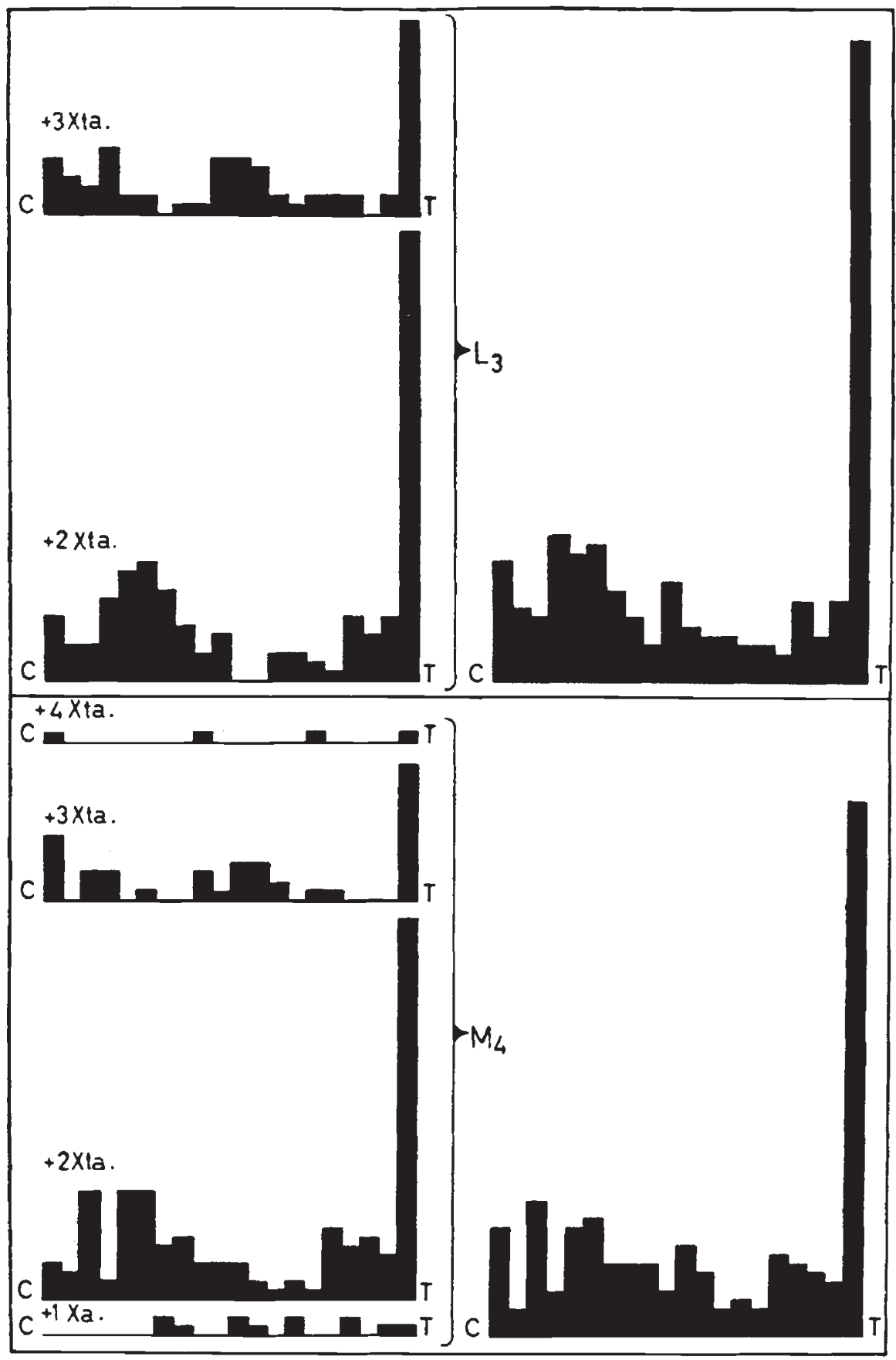

FIG. 5-6.-Chiasma distributions for the $\mathrm{L}_{3}$ and $\mathrm{M}_{4}$ bivalents. 
and more despiralised at zygotene and pachytene it can form more chiasmata, the same interference distance operating over an effectively longer chromosome. But this, of course, is the complete inverse of what one expects on a torsional theory of chiasma formation. An alternative explanation is that a chiasma produces some relaxation of chromosome coiling (despiralisation) or that it partly inhibits coiling at diplotene. In this case, greater despiralisation or coiling retardation would be found in bivalents possessing high chiasma frequencies than in those in which the number of chiasmata present was low.

Thus far then, the findings complicate rather than clarify the issue of chiasma distribution and are apparently contrary to previous ideas in this field. Further information on what is taking place however, is obtained if the original ' $d$ ', ' $i$ ' and ' $r$ ' measurements, expressed as percentages, are plotted in histogram form, for each bivalent at each chiasma frequency found. In addition, a composite histogram may be prepared showing the overall chiasma distribution for that bivalent. The histograms so obtained are provided in figs. 3-13. Percentages must necessarily be used for this purpose in order to eliminate the effects of chromosomes in different cells being at different degrees of contraction.

These figures show that chiasma formation takes place along the entire length of most chromosomes, but clustered distributions are present around the positions where most chiasmata form. These are roughly symmetrical for interstitial chiasmata, while the distributions of the most proximal chiasmata are somewhat more variable. The most conspicuous distribution on each bivalent, however, is that of the distal chiasma. In almost every bivalent this shows a markedly skew distribution of considerable magnitude.

According to the theory of chiasma distribution suggested by Mather, one would expect the position and distribution of the firstformed chiasma to be bivalent-specific, while the distributions of subsequent chiasmata should be similar and comparable ' $i$ ' distances apart in all chromosome classes. Now if a linear chiasma frequency/ chromosome length relationship is to be obtained on rising from the minimum chiasma frequency level of one per bivalent, both ' $d$ ' and ' $i$ ' distances must be constant for all members of the complement. If either the ' $d$ '. or ' $i$ ' distance alone or both were variable, a nonlinear relationship would be obtained. If either varied in an uncorrelated manner, the line would zig-zag, while if the variation was correlated and progressively increased or decreased with chromosome length, the regression would be curvilinear. It would be concave if a decrease was involved and convex if an increase was involved (fig. I4).

Assuming a linear relationship one could therefore construct a diagram, to scale, incorporating the ' $d$ ' and ' $i$ ' distances, indicate below it the lengths of the different members of the complement and then be able to see from the guiding distributions above, both the 


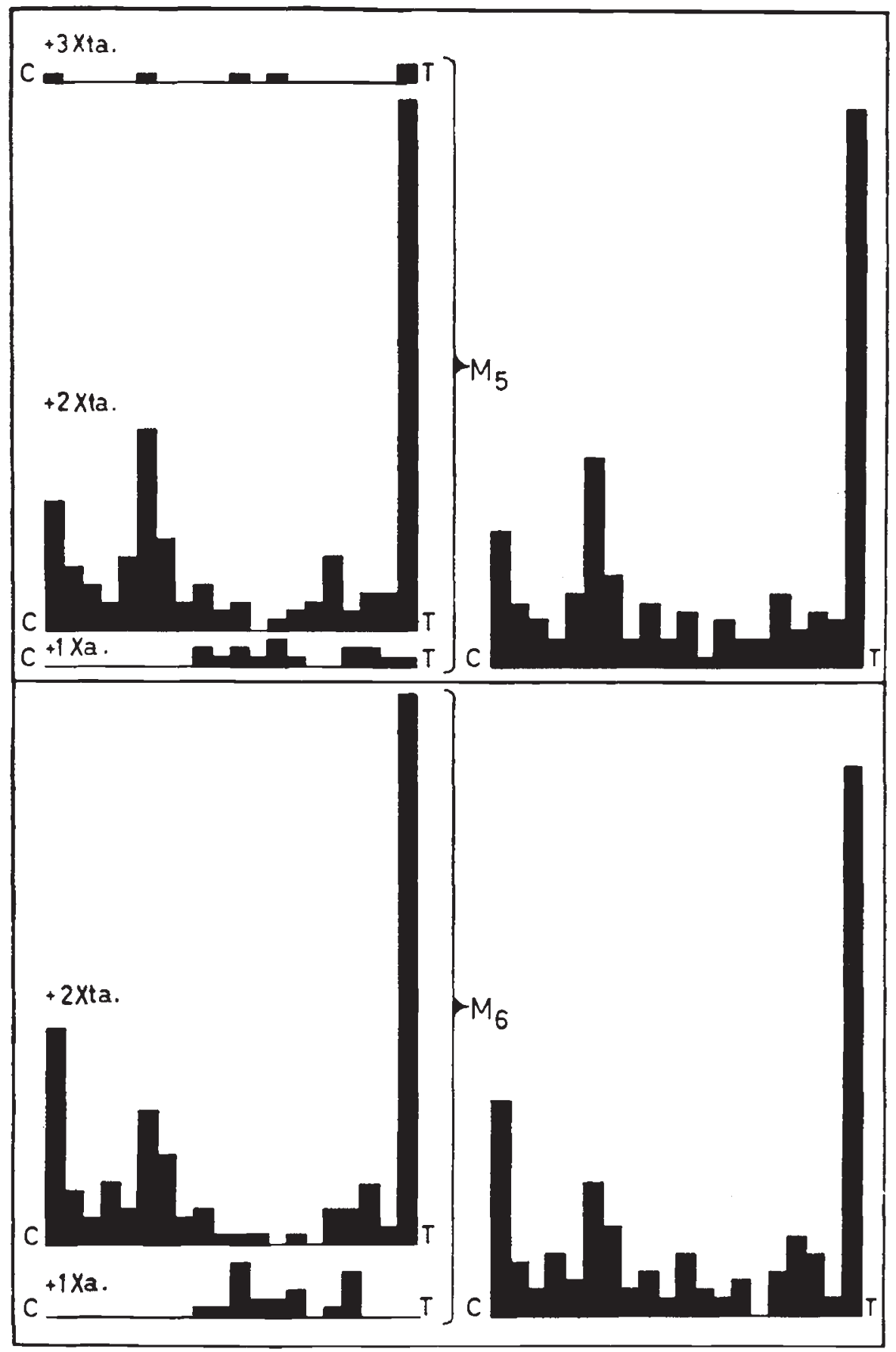

Figs. 7-8.-Chiasma distributions for the $\mathrm{M}_{5}$ and $\mathrm{M} 6$ bivalents. 


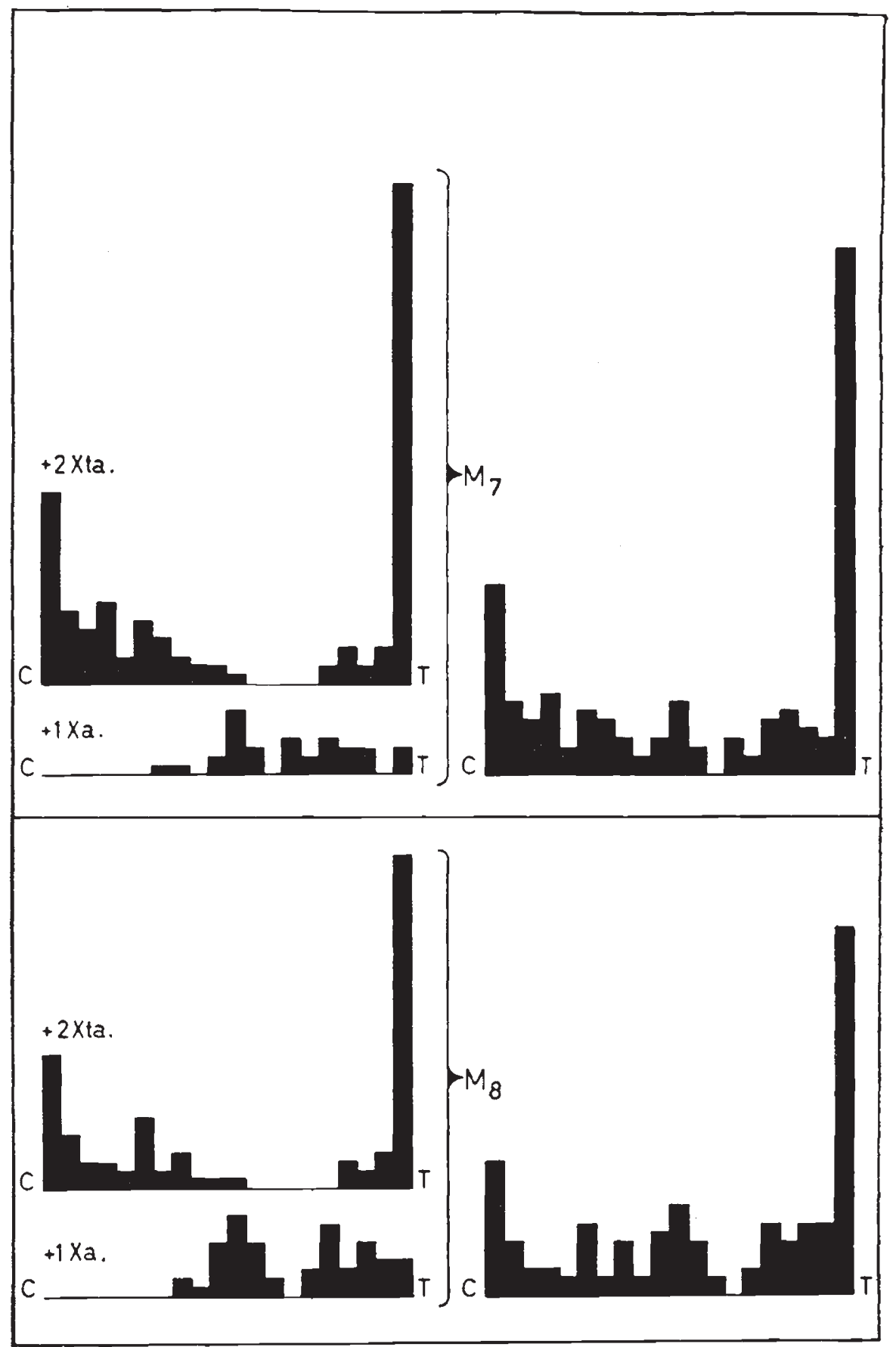

FIgs. 9-10.-Chiasma distributions for the $\mathrm{M}_{7}$ and $\mathrm{M} 8$ bivalents. 


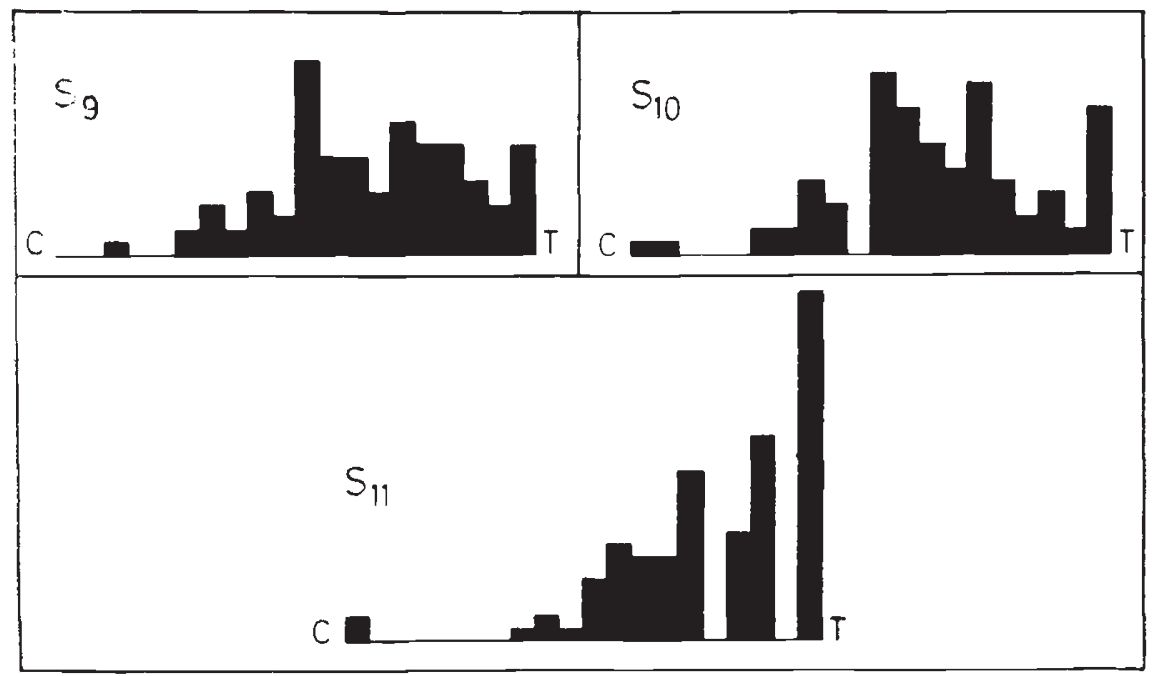

Figs. 11-13.-Chiasma distributions for the $S_{9}, S_{10}$ and $S_{11}$ bivalents. Only a composite histogram is provided.

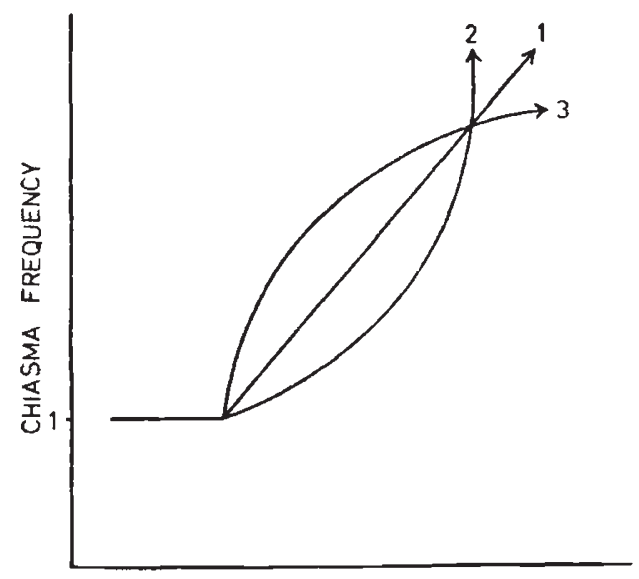

CHROMOSOME LENGTH

Fic. 14.-Theoretical chiasma frequency/chromosome length relationships for various combinations of ' $d$ ' and ' $i$ '. On rising from the minimum chiasma frequency level of one per bivalent a linear relationship ( 1 ) would be obtained if both ' $d$ ' and ' $i$ ' remained constant throughout the complement. If there was a progressive increase or decrease of either distance with increase of chromosome length the result would be curvilinear. It would be concave (2) if there was a progressive decrease of either distance individually or if both decreased together. It would be convex $(3)$ if either, or both, increased. It would zig-zag if variation in either distance was uncorrelated.

chiasma frequency for that length of chromosome and the approximate shape of the chiasma distributions it should possess (fig. I5). The first-formed distribution ought to be comparable in all bivalents, while the relative position and completeness of the last-formed distribution would depend on the length of the bivalent in question. Furthermore, bivalents of similar length should clearly possess similar distributions. 
Comparing each of the composite histograms obtained for Schistocerca gregaria (figs. $3-15$ ) it is seen that there is no clear correspondence of distributions if the first chiasma is considered to form near the centromeric end of the chromosome. If, on the other hand, one assumes that chiasma formation in this species does not start near the centromere but at the distal end and proceeds along the chromosome towards the centromere, the distributions look much more promising.

If the first chiasma forms at, or near, the distal tip of the chromosome, with a ' $d$ ' distance near, or equal to, zero, this immediately

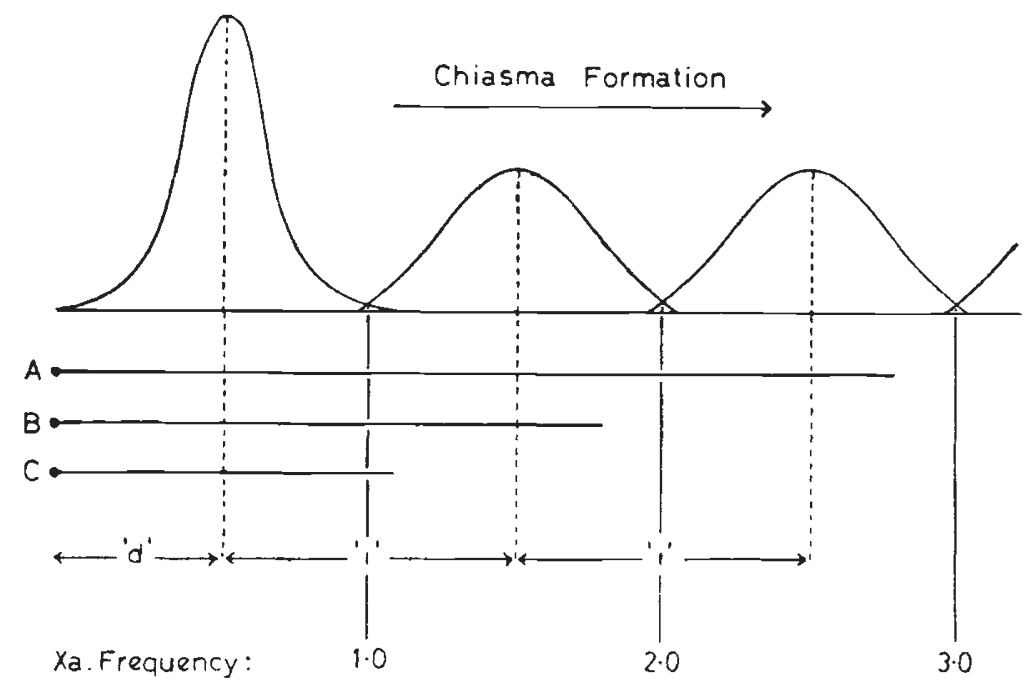

FiG. I5.-Diagram to illustrate the theoretical distribution of chiasmata along chromosomes of different lengths ( $A, B, C)$ under the operation of a constant differential (' $d$ ') and interference (' $\mathrm{i}$ ') distance. Black circles mark centromeric position.

clarifies the skew distribution of great height exhibited by this chiasma in each bivalent, for a distally located chiasma is almost invariably present in all but the S-bivalents (which tend to fall in a class of their own). Furthermore, when only one chiasma forms, in this species, it is usually confined to the distal half of the chromosome, not the proximal. Subsequent chiasmata should fall a mean of ' $i$ ' distances apart and, depending on chromosome length, one should obtain all or part of a symmetrical chiasma distribution at the centromeric end (i.e. the reverse of fig. I5).

If, in fact, this is the situation obtaining in this species, it should be possible to construct a diagram similar to fig. 15 using the operative ' $\bar{l}$ ' distance and $\mathrm{a}$ ' $\overline{\mathrm{d}}$ ' distance of zero. On placing the chromosome lengths for all members of the complement below this figure one ought to be able to read off, according to chromosome length, both chiasma frequency and the kind of chiasma distribution one should obtain. 
Care must, however, be exercised in selecting the overall ' $\bar{i}$ ' distance. This will be made evident on consulting fig. 15 once more. Thus, a small chromosome which usually forms only one chiasma may rarely form two if it is long enough to reach the tail of the ' $i$ ' distance distribution. However, the mean ' $i$ ' distance obtained from such a bivalent will clearly not be a valid estimate of the operative ' $\overline{1}$ ' distance as such : it will be a gross underestimate. Indeed, the ' $\bar{i}$ ' obtained from any bivalent with a chiasma frequency of less than two per bivalent will be too small. This explains at least one of the causes of the considerable variation obtained in the mean ' $i$ ' distances shown in tables $I$ and 2.

TABLE 2

Overall mean ' $\bar{d}$ ', ' $\bar{i}$ ' and ' $\bar{r}$ ' measurements in micra, for each of the II bivalents of Schistocerca gregaria, irrespective of chiasma frequency

\begin{tabular}{|c|c|c|c|c|c|c|c|c|}
\hline \multirow{2}{*}{$\begin{array}{l}\text { Chromo- } \\
\text { some }\end{array}$} & \multicolumn{2}{|c|}{$\cdot \bar{d}}$, & \multicolumn{2}{|c|}{; } & \multicolumn{2}{|c|}{$\dot{r}}$, & \multicolumn{2}{|c|}{ Mean length } \\
\hline & $\bar{x}$ & $\mathrm{~V}_{\bar{x}}$ & $\bar{x}$ & $\mathrm{~V}_{\bar{x}}$ & $\overline{\boldsymbol{x}}$ & $\mathrm{V}_{\bar{x}}$ & $\overline{\boldsymbol{x}}$ & $\mathrm{V}_{\bar{x}}$ \\
\hline $\mathrm{L}_{1}$ & $3 \cdot 16$ & 0.052 & $7 \cdot 58$ & 0.027 & 0.88 & 0.017 & $18 \cdot 37$ & 0.212 \\
\hline $\mathrm{L}_{2}$ & $2 \cdot 6 \mathrm{I}$ & 0.032 & $7 \cdot 08$ & $0.03^{8}$ & $1 \cdot 26$ & 0.017 & 14.99 & 0.109 \\
\hline $\mathrm{L}_{3}$ & $2 \cdot 83$ & 0.035 & $7 \cdot 23$ & 0.053 & 0.64 & 0.012 & $12 \cdot 31$ & 0.095 \\
\hline $\mathrm{M}_{4}$ & $2 \cdot 98$ & 0.058 & $6 \cdot 12$ & $0.04^{2}$ & $1-01$ & 0.022 & $10 \cdot 36$ & $0.05^{8}$ \\
\hline $\mathrm{M}_{5}$ & $2 \cdot 42$ & 0.034 & 5.62 & $0.04^{1}$ & 0.85 & 0.019 & $8 \cdot 06$ & 0.034 \\
\hline M6̆ & 2.01 & 0.035 & $5 \cdot 39$ & 0.025 & 0.80 & 0.016 & 7.07 & 0.027 \\
\hline $\mathrm{M}_{7}$ & $2 \cdot 07$ & 0.038 & $5 \cdot 13$ & 0.029 & $0.7^{8}$ & 0.013 & $6 \cdot 29$ & 0.021 \\
\hline M8 & $2 \cdot 85$ & 0.034 & 4.71 & 0.024 & 0.96 & 0.012 & 5.57 & 0.016 \\
\hline $\mathrm{S}_{9}$ & $2 \cdot 60$ & 0.012 & $(6 \cdot 00)$ & $\ldots$ & $1 \cdot 35$ & 0.009 & 4.06 & 0.011 \\
\hline Sio & $2 \cdot 09$ & 0.008 & $(6 \cdot 00)$ & $\ldots$ & 1.19 & 0.006 & $3 \cdot 36$ & 0.006 \\
\hline$S_{11}$ & $2 \cdot 25$ & 0.005 & $(4 \cdot 00)$ & $\ldots$ & 0.65 & 0.005 & 2.94 & 0.004 \\
\hline
\end{tabular}

The first accurate estimate of the overall ' $i$ ' distance should thus, theoretically, be obtained from a chromosome with a consistent mean chiasma frequency of, or just over, two chiasmata per bivalent. In this complement the $M_{4}$ falls into this category and its ' $i$ ' distance is $6.12 \mu$ at diplotene. However, this too is an underestimate for a different reason, which will be explained more fully in a moment (see p. I89). The best estimate of the ' $\bar{i}$ ' distance is provided by the L-chromosomes, which have mean chiasma frequencies of well over two per bivalent. These chromosomes avoid both sources of error present in ' $\bar{i}$ ' estimates provided by smaller members of the complement. The ' $\bar{i}$ ' obtained from these three chromosomes is $7.3 \mu$ at diplotene.

A diagram may now be constructed using the ' $i$ ' distance so obtained and $a$ ' $\bar{d}$ ' distance of zero, assuming the chiasma formation starts at the distal end and proceeds along each bivalent towards the centromere (fig. I6). A very close fit to the expected pattern is obtained, but there are a few differences. The proximal chiasma 
does not always show the distribution one would expect from fig. 16 . Furthermore, the M-chromosomes have a slightly higher chiasma frequency than they should. Clearly the situation is somewhat more complex than provided in our initial hypothesis.

The regression of chiasma frequency per bivalent on chromosome length supports this contention. On rising from the minimum chiasma frequency level of one per bivalent, the relationship is not linear throughout, but possesses a median flexure near the $2 \cdot 0$ chiasmata per bivalent level (fig. 17). Though the curve is linear above the two chiasmata level, the $\mathrm{M}_{5}, 6,7$ and 8 bivalents consistently possess higher chiasma frequencies than they should if an overall linear relationship is to be obtained. In this figure the chromosome lengths used are those obtained from the diplotene cells measured in this

TABLE 3

Chiasma frequencies at diplotene in Schistocerca gregaria

(Based on the scoring of 25 cells at diplotene from each of ten individuals.)

\begin{tabular}{|c|c|c|c|c|c|c|c|c|c|c|c|c|}
\hline \multirow{2}{*}{ Item } & \multicolumn{11}{|c|}{ Chiasma frequency per bivalent } & \multirow{2}{*}{$\begin{array}{l}\text { Cell } \\
\text { total }\end{array}$} \\
\hline & $\mathrm{LI}_{\mathrm{I}}$ & $\mathrm{L}_{2}$ & $\mathrm{~L}_{3}$ & $M_{4}$ & $M_{5}$ & M6 & $M_{7}$ & M8 & S9 & Sro & $S_{I I}$ & \\
\hline$\overline{\boldsymbol{x}}$ & 3.04 & $2 \cdot 60$ & $2 \cdot 31$ & $2 \cdot 05$ & $1 \cdot 92$ & $I \cdot 85$ & $r \cdot 69$ & $1 \cdot 34$ & $1 \cdot 00$ & $1 \cdot 00$ & $\mathrm{I} \cdot 00$ & $19 \cdot 8 x$ \\
\hline $\mathbf{V}_{\overline{\boldsymbol{x}}}$ & 0.002 & 0.003 & $0 \cdot 004$ & $0 \cdot 003$ & $0 \cdot 003$ & 0.002 & 0.001 & 0.003 & 0.000 & $0 \cdot 000$ & 0.000 & $0 \cdot 109$ \\
\hline
\end{tabular}

investigation, in preference to mitotic lengths. These are used for two reasons. First the chromosomes are very much longer at diplotene and can therefore be measured with greater accuracy. Secondly, the regression of diplotene length on mitotic length is not linear, but curvilinear : there is not an exact correspondence between the lengths of different members of the complement at mitotic metaphase and at meiotic prophase (fig. 18). Chromosome length is a very difficult character to fix unambiguously, for it is influenced by a number of variables. Because measurement and identification is more accurate at diplotene and because the diplotene lengths fit the chiasma frequency expectations best these are considered to be the most reliable to use at the moment.

How then are we to explain the non-linear middle segment of the curve? Why do the M-chromosomes possess higher chiasma frequencies than they should and why, once we pass the point where a minimum of two chiasma per bivalent are consistently formed, should the effect almost disappear and a linear relationship obtain? There are three possible explanations which could, in theory, produce such effects :

(i) That the ' $d$ ' distance is of lower magnitude in the M-chromosomes than in the L-'s and that there is a sudden shift in its magnitude 
in chromosomes capable of exceeding the level of two chiasmata per bivalent. This possibility, difficult to conceive in mechanism, is ruled out by the close approximation of the ' $\mathrm{d}$ ' distance, in all chromosomes, to zero.

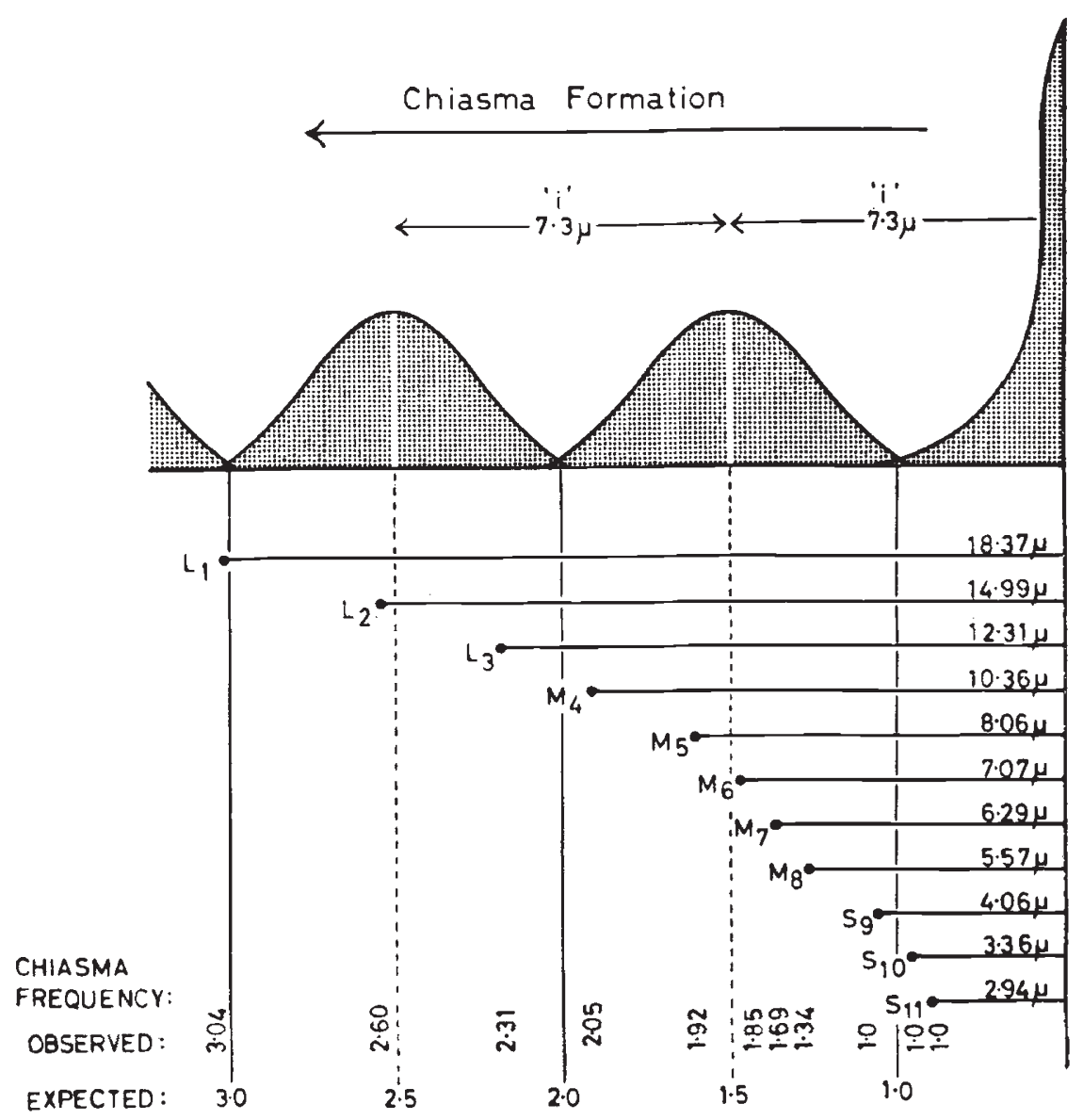

Fig. 16.-Diagram to illustrate the distribution of chiasmata along the eleven bivalents (LI-SI 1$)$ of $S$. gregaria, assuming that chiasma formation starts at the distal end of all bivalents, with a ' $d$ ' distance of near zero and an ' $i$ ' distance of 7 ' $3 \mu$ (diplotene length). The chiasma frequencies observed at diplotene for each bivalent are provided for direct comparison with those expected. In addition, the diplotene length of each chromosome is provided in $\mu$. The centromeric positions are again marked by black circles.

(ii) That the ' $i$ ' distance varies in the manner specified above for the ' $\mathrm{d}$ ' distance. This is, of course, a possibility, but an unlikely one. Why, or how, the interference distance should be bivalentspecific at the M-chromosome level and then for no apparent reason change, once the length of Io $\mu$ (diplotene length) is exceeded, becoming both larger and similar for all longer chromosomes, is difficult to understand. The most likely explanation is the third possibility. 
(iii) That chiasma formation may, on occasion at least, start simultaneously at both ends of a chromosome and proceed towards the middle. Clearly, two such chiasmata cannot invariably form at the same time, otherwise all bivalents would possess chiasma frequencies of over two per bivalent. But all of the observed deviations can be explained if this takes place $30-40$ per cent. of the time for all bivalents (the smalls excepted). This would raise the chiasma frequencies of the $\mathrm{M}_{5-8}$ bivalents to their present levels, from that expected on a linear relationship. However, once the length of a chromosome was

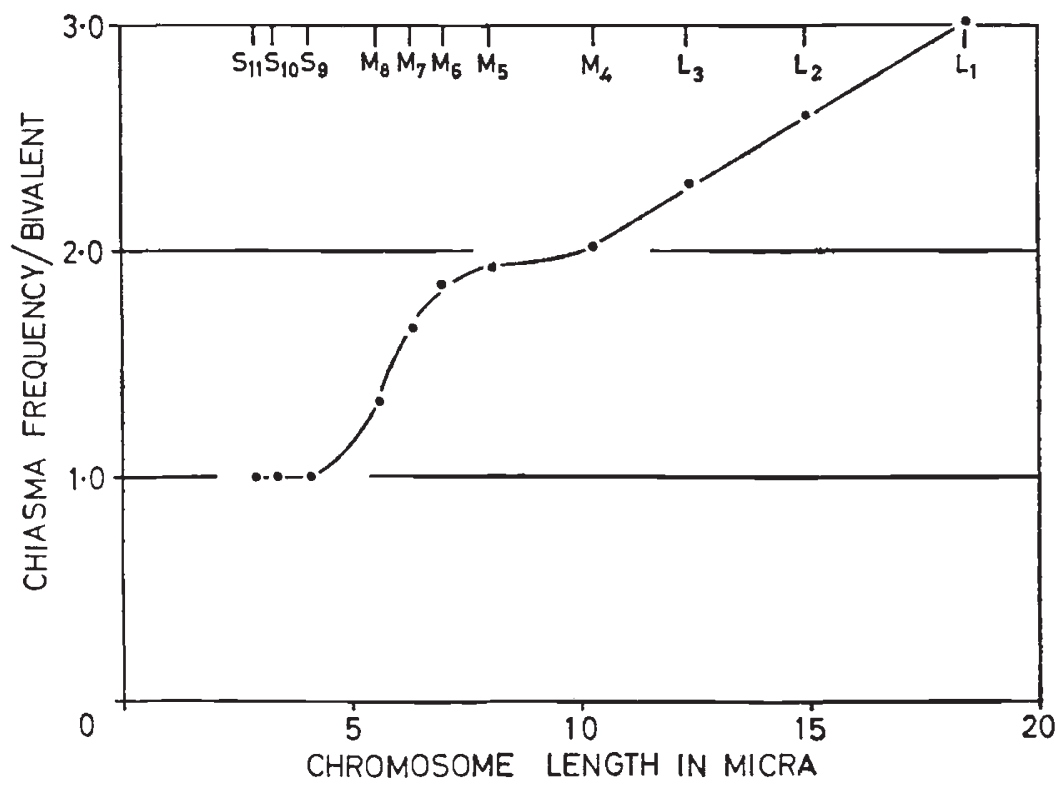

Frg, 17.-Chromosome length/chiasma frequency relationship for $S$. gregaria. A median flexure is present near the $2 \cdot 0$ chiasmata per bivalent level, above which the regression is linear. The chiasma frequency values used in this fig. were obtained from ro individuals, from each of which 25 cells were scored.

sufficiently in excess of the interference distance for it consistently to form two chiasmata, then the effect should be lost. For whether the two chiasmata form in a linear sequence under the operation of the interference distance, or whether they form simultaneously, the magnitude of their interference then usually excluding the formation of a third between them, the end result should be similar. However, the elevating effect of this process is not completely absent from the $\mathrm{M}_{4}$, nor cven from the $\mathrm{L}_{3}$ and $\mathrm{L}_{2}$, for their chiasma frequencies are very slightly in excess of expectation (figs. 16 and 17 ). This is presumably due to the occasioi.... formation of a third chiasma in the median chromosome segment, where interference from the two terminal chiasma will be minimal (fig. I9). This, when it took place, would produce an equidistant spacing of chiasmata and accentuate, to the level found in practice, the equidistant spacing which would normally be expected on a linear sequence of chiasma formation. 
This near simultaneous formation of two chiasmata, one at either end of a chromosome, is therefore responsible for the two confusing issues mentioned earlier. First, it produces the anomalous distributions of chiasma position obtained at the centromeric end of some

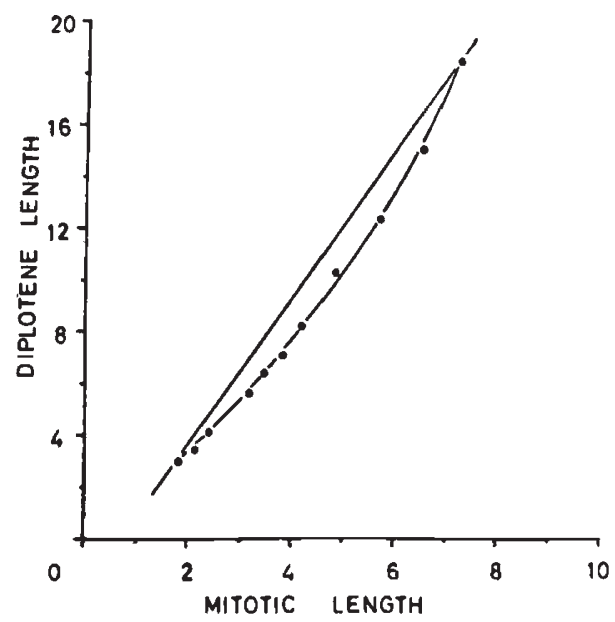

FIG. 18.-Regression of diplotene length on mitotic length for the eleven chromosome pairs of S. gregaria. The diplotene lengths are those obtained in this investigation (table 2). The mitotic lengths are those of John and Naylor (196r).

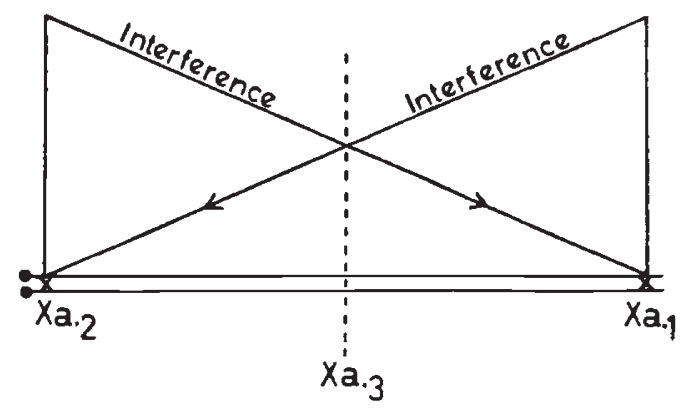

FIG. 19.-Diagram to illustrate the restriction of a third chiasma to the median region of a chromosome arm under the combined interference of two terminally formed chiasmata. Equidistant spacing results. Black circles again indicate centromeric position.

chromosomes (e.g. the $\mathrm{M}_{5}$ and $\mathrm{M} 6$ ) and secondly, it results in an apparently smaller interference distance being present between the two so-formed terminal chiasmata of an M-bivalent. This then is the second source of error in the ' $\bar{i}$ ' distance values obtained for M-chromosomes.

\section{CONCLUSION AND SUMMARY}

In conclusion, the information obtained in this diplotene investigation on the locust Schistocerca gregaria supports the contention that chiasma formation usually takes place in a linear sequence, 
starting from a fixed point, and that the interference (' $i$ ') distance along paired homologues is approximately constant throughout the complement. In this species chiasma formation usually begins at the distal end of each acrocentric chromosome, with a constant differential (' $d$ ') distance equal to or near zero. The overall interference distance, as measured at diplotene, is $c .7 \cdot 3 \mu$. An additional complicating factor is present in this species, however, which confuses interpretation : sometimes chiasma formation apparently begins at both ends of a chromosome and proceeds towards the middle. This must take place at a frequency of $30-40$ per cent. to explain the observed deviations. However, even in these cases, the same overall interference distance would appear to be in operation. This complication results in the chiasma frequency/chromosome length relationship being non-linear for M-chromosomes, though linear above the level of two chiasmata per bivalent.

Acknowledgments.-I would like to express my gratitude to Professor K. Mather and $\mathrm{Dr} \mathrm{B}$. John for the encouragement and assistance they have given me.

\section{REFERENCES}

Belling, J. 1931. Chiasmas in flowering plants. Univ. Calif. Pub. Bot., i6, 31 1-338. BELLING, J. 1933. Crossing-over and gene rearrangements in flowering plants. Genetics, $18,388-412$.

DARLington, C. D. 1935a. The time, place and action of crossing-over. F. Genet., $31,185-212$.

DARLington, c. D. 1935b. The internal mechanics of the chromosomes. Proc. Roy. Soc. $B, 118,33-96$.

haldane, J. B. s. I931. The cytological basis of genetical interference. Cytologia (Tokyo), 3, 54-65.

HENDERSON, s. A. 1962. Temperature and chiasma formation in Schistocerca gregaria. II. Cytological effects and the mechanism of heat-induced univalence. Chromosoma (Berl.) I3, 437-463.

HeNDERSON, S. A. 1963. Temperature and chiasma formation in Schistocerca gregaria. I. An analysis of the response at a constant $40^{\circ} \mathrm{C}$. Heredity, 18, 77-94.

JOHN, B., AND HENDERson, S. A. 1962. Asynapsis and polyploidy in Schistocerca paranensis. Chromosoma (Berl.), 13, $111-147$.

JOHN, B., AND NAYLOR, B. I961. Anomalous chromosome behaviour in the germ line of Schistocerca gregaria. Heredity, $16,187-198$.

MATHER, K. 1936. The determination of position in crossing-over. I. Drosophila melanogaster. F. Genet., 33, 207-235.

MATHER, K. 1937. The determination of position in crossing-over. II. The chromosome length-chiasma frequency relation. Cytologia (Tokyo) Fujii Jub. Vol. 514-526.

MATHER, K. 1938. Crossing-over. Biol. Rev., 13, 252-292.

MATHER, K. 1940. The determination of position in crossing-over. III. The evidence of metaphase chiasmata. F. Genet., 39, 205-223.

MULleR, H. J. 1916. The mechanism of crossing-over. Amer. Nat., 50, 193-221, 284-305, 350-366, 42 I-434.

PRITCHARD, R. H. 196o. The bearing of recombination analysis at high resolution on genetic fine structure in Aspergillus nidulans and the mechanism of recombination in higher organisms. Microbial genetics. Symp. Soc. Gen Microbiol., Io, $155-180$. 